\title{
PERFORMANCE OF RECALIBRATION SYSTEMS FOR GCM FORECASTS FOR SOUTHERN AFRICA
}

\author{
MXOLISI E. SHONGWE, ${ }^{\mathrm{a}, \mathrm{b}, *}$ WILLEM A. LANDMAN ${ }^{\mathrm{b}, \mathrm{c}}$ and SIMON J. MASON ${ }^{\mathrm{d}}$ \\ a Swaziland National Meteorological Service, Mbabane, Swaziland \\ ${ }^{\mathrm{b}}$ Department of Geography, Geoinformatics and Meteorology, University of Pretoria, South Africa \\ ${ }^{\mathrm{c}}$ South African Weather Service, Pretoria, South Africa \\ d International Research Institute for Climate and Society, Earth Institute of Columbia University, New York, USA
}

Received 23 August 2005

Revised 6 January 2006

Accepted 12 January 2006

\begin{abstract}
Two regression-based methods that recalibrate the ECHAM4.5 general circulation model (GCM) output during austral summer have been developed for southern Africa, and their performance assessed over a 12-year retroactive period 1989/90-2000/01. A linear statistical model linking near-global sea-surface temperatures (SSTs) to regional rainfall has also been developed. The recalibration technique is model output statistics (MOS) using principal components regression (PCR) and canonical correlation analysis (CCA) to statistically link archived records of the GCM to regional rainfall over much of Africa, south of the equator. The predictability of anomalously dry and wet conditions over each rainfall region during December-February (DJF) using the linear statistical model and MOS models has been quantitatively evaluated. The MOS technique outperforms the raw-GCM ensembles and the linear statistical model. Neither the PCR-MOS nor the CCA-MOS models show clear superiority over the other, probably because the two methods are closely related. The need to recalibrate GCM predictions at regional scales to improve their skill at smaller spatial scales is further demonstrated in this paper. Copyright (C) 2006 Royal Meteorological Society.
\end{abstract}

KEY WORDS: seasonal climate prediction; model output statistics; canonical correlation analysis; principal components regression; general circulation models; model validation; sea-surface temperatures; climate variability; southern Africa

\section{INTRODUCTION}

The irregular recurrence of anomalous climate events such as droughts and floods demonstrates the urgent need for the enhancement of accurate seasonal to interannual climate monitoring and prediction information in the Southern African Development Community (SADC) region. Most of the national economies within the region are highly dependent on agriculture and hence are vulnerable to year-to-year fluctuations in the amount and distribution of precipitation. Following the 1991-1992 El Niño related drought, and its adverse effects, understanding and techniques behind climate forecasting have developed rapidly (Hastenrath et al., 1995; Barnston et al., 1996; Mason et al., 1996; Makarau and Jury, 1997; Rocha and Simmonds, 1997; Mason, 1998; Tennant, 1999; Landman and Mason, 1999b; Landman and Tennant, 2000; Landman et al., 2001; Unganai and Mason, 2002; Landman and Goddard, 2002). The progress being made in exploring prospects for seasonal climate prediction over the region using techniques of varying complexity is encouraging.

Earlier approaches to climate forecasting involved the use of purely statistical methods such as multiple linear regression (Mason et al., 1996; Makarau and Jury, 1997), discriminant analysis (Mason, 1998), canonical correlation analysis (CCA) (Barnston et al., 1996; Landman and Mason, 1999b), and neural networks (Hastenrath et al., 1995). Statistical climate forecasting techniques rely on empirical relationships between

\footnotetext{
* Correspondence to: Mxolisi E. Shongwe, Royal Netherlands Meteorological Institute, P.O. Box 201, 3730 AE De Bilt, The Netherlands; e-mail: shongwe@knmi.nl
} 
climate variables to give an indication of the expected regional climate anomalies based on prevailing largerscale climate patterns. Their success is therefore dependent on the amount and quality of available historical data. Observational surface data is available for much of SADC, save for a few countries that have been victims of civil wars and political volatility, creating prospects for successful application of statistical tools in seasonal climate forecasting. Statistical methods, particularly those that are linear, are often preferred because they are relatively simple and cheap to develop. However, the predictive skills of these models are limited owing to the exclusion of non-linear relationships among variables. Statistical methods are trained using historical data such that past outcomes are averaged with an inherent inability to distinguish one situation from generic events. For instance, linear relationships amongst climate variables have been found not to be always stable (Landman and Mason, 1999a). Linear statistical models are unable to account for such instabilities in the link among climate variables. Furthermore, most statistical methods inherently assume multivariate normality among the populations of the variables, an assumption that may be invalid.

Noting that much of the potential of statistical models has already been realised, development efforts are now shifting towards more sophisticated numerical models that represent the physics of the climate system. Theoretically, numerical models of the climate system should offer a possible solution to the deficiencies of statistical models. Since general circulation models (GCMs) are based on the physical laws governing the processes within the climate system, with improved and thorough representation of the real physics, there is room for further improvement in forecast skill. GCMs have demonstrated skill in simulating large-scale atmospheric features in the southern African region (Joubert, 1997), but are not equally skilful at much smaller spatial scales. The perceived failure of climate models on the local scale can be attributed in part to the coarse horizontal resolution whereby the structure of the earth's surface is not adequately described in the models. The use of spatially uniform parameterisation schemes for different locations in the world could produce additional errors in GCM simulations. Unfortunately, for most climate forecast applications such as in agriculture and water resources, it is the subgrid scale information that is useful. There is therefore a need to recalibrate or downscale GCM output by relating large-scale information from climate models to regional or local climate parameters.

The downscaling methods found in the literature vary in their technical complexity. Dynamical downscaling approaches include using finer GCM resolutions or nesting a limited area model (LAM) within a GCM (Kidson and Thompson, 1998; Murphy, 1999, 2000). However, both methods are computationally intensive and expensive, and hence are restricted to institutions with sufficient computational power. A relatively easy and cheap approach is statistical downscaling whereby relationships between GCM simulated large-scale fields and regional rainfall is derived empirically. Several statistical downscaling techniques of varying complexity are being applied. Zorita and von Storch (1999) give a review of some of the statistical methods in use.

In this study, two regression-based recalibration methods are applied and the operational levels of their forecast skill are assessed using a retroactive forecast procedure (Landman et al., 2001). The methods considered are CCA and principal components regression (PCR). CCA has been widely applied in downscaling coarsely gridded climate data from GCMs to a finer spatial resolution or to recalibrate GCM output to specifically defined rainfall regions (Karl et al., 1990; von Storch et al., 1993; Zorita and von Storch, 1999; Landman and Tennant, 2000; Busuioc et al., 1999, 2001; Landman and Goddard, 2002), but PCR has been less frequently applied (Mo and Stratus, 2002) despite its procedure being simpler. The performance of the recalibration systems is further compared with that of raw-GCM ensembles and a simple linear statistical model. These forecasting models provide a baseline of skill for the recalibration methods that is more difficult to beat than much simpler schemes such as persistence forecasts.

\section{DATA AND METHODOLOGY}

\subsection{Rainfall data and homogenous regions}

Monthly rainfall data for the 1961-2001 period were obtained for 255 stations distributed across 11 countries within the SADC region. Station locations are denoted by dots in Figure 1(a). The distribution of the stations is markedly irregular: most stations are concentrated over the central and southern parts with very 
(a) GCM grids and stations

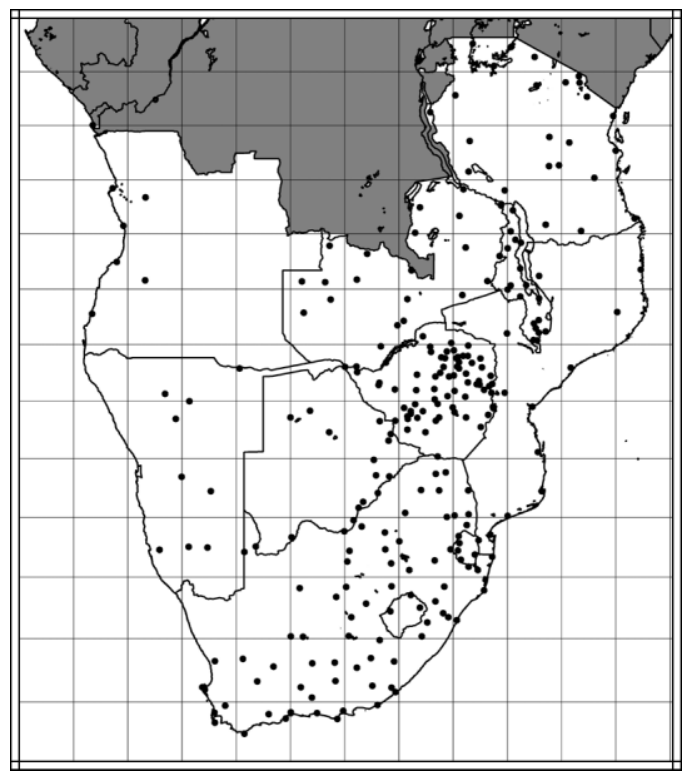

(b) Rainfall zones

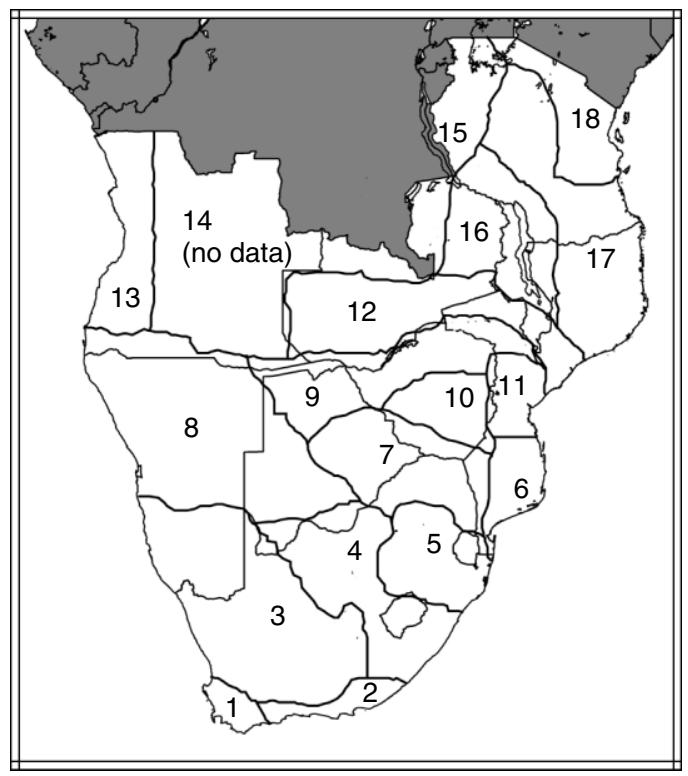

Figure 1. (a) Location maps of the 255 rainfall stations and the ECHAM4.5 GCM grid boxes; (b) 18 regions obtained from cluster analysis. Countries where data were unavailable are shaded. The map covers an area from the Equator to $36^{\circ} \mathrm{S}$ and about $8{ }^{\circ} \mathrm{E}$ to $42^{\circ} \mathrm{E}$

few in the west. The rainfall data were obtained from the SADC Drought Monitoring Centre, where they undergo quality control. Only stations with less than $5 \%$ missing values were selected. Missing values were replaced using a regression method from the highest correlated neighbouring station (Tabachnick and Fidell, 2001).

Homogeneous rainfall regions have been defined by grouping the rainfall stations on the basis of spatial rainfall patterns that occur during the main rainfall season (October-March) over southern Africa using cluster analysis (Mason, 1998; Mimmack et al., 2001). The aim of clustering the stations was to define regions within 
which the processes responsible for seasonal to interannual rainfall variability are similar. The clustering was based on Euclidean distances calculated from the unstandardised principal component scores (Mimmack et al., 2001). The number of retained principal components varied between 10 and 20. The higher number of principal components was retained to ensure that small but distinct regions are identified. A total of 18 regions have been defined as shown in Figure 1(b). No data were available over eastern Angola (region 14); hence this region was not considered in the analysis. Notably, the clustering did not show distinct regions along the eastern coastal regions of South Africa, inconsistent with findings by previous authors (Mason, 1998; Landman et al., 2001) who considered a much smaller domain within the subregion than is used here. The differences from earlier regionalisations are a result of the use of fewer stations over the same areas in the present study, and the fact that the clustering was based only on seasonal rather than annual rainfall totals.

Three-month overlapping totals were calculated within the main rainfall season (October-March) over southern Africa. The rainfall totals for each 3-month period were standardised by subtracting the mean and dividing by the standard deviation, and then averaged across all the stations in each region. OND totals were calculated for the period 1961 to 2000, NDJ and DJF for the period 1961/62 to 2000/01, and JFM for the period 1962 to 2001. Over much of SADC, December-January constitutes the peak summer season and is considered most important to agriculture, and economic welfare of society. For this reason, only results for DJF are presented in this paper.

\subsection{Sea-surface temperatures}

Although incorporating alternative variables into the predictor set may effect improvements in the forecast skill of statistical models (Hastenrath et al., 1995; Makarau and Jury, 1997), sea-surface temperatures (SSTs) have remained the most widely used predictors for southern African summer rainfall (Barnston et al., 1996; Mason, 1998; Landman and Mason, 1999b). In this study, near-global $\left(60^{\circ} \mathrm{N}-60^{\circ} \mathrm{S}\right)$ extended reconstructed SSTs version 2 (Smith and Reynolds, 2004) are the sole predictors used for developing and running the empirical-statistical model. The SST data on a $2 \times 2^{\circ}$ lat/lon grid were extracted for the period May 1961 to November 2000.

Three-monthly SST averages were calculated such that the lead time is 1 or 2 months for each target seasonal rainfall period. For example, the ASO (JAS) average SSTs were used to derive an empirical relationship with DJF standardised rainfall resulting in a 1-2 month(s) lead time. The training period was varied for each retroactive forecast period (discussed in Section 2.6). For example, JAS 1961-1988 SST and DJF 1961/62-1988/89 rainfall data was used to fit a CCA model, which was then used to predict DJF 1989/90-1991/92 rainfall. The training period was updated to yield predictions for each 3-year retroactive period up to DJF 2000/01. A similar procedure was repeated using the ASO average SSTs.

\subsection{The ECHAM4.5 GCM}

The ECHAM4.5 GCM is a modified version of the ECHAM4 GCM (Roeckner et al., 1996). The hindcast mode of the GCM consisting of 12 ensemble members is used in this study. In this mode, the observed monthly SST anomalies (serving as boundary forcing for the GCM) persisted on top of the monthly varying seasonal cycle of SSTs for the season (3-months) of interest. The GCM predictions from this mode are available at 2 months or shorter lead times. To make a suitable comparison with the linear statistical model, the 2- and 1-month lead predictions are considered. The model grids at T42 (about $2.8^{\circ}$ lat/lon) horizontal resolution over an area of approximately $0^{\circ}$ to $36^{\circ} \mathrm{S}$ and $8^{\circ} \mathrm{E}$ to $42^{\circ} \mathrm{E}$ are shown in Figure 1(a). The model rainfall predictions have been averaged over all grid points located within each rainfall region (Figure 1(b)).

For each training period and region, the model climate has been defined on the basis of its predictions from the preceding years. These predictions have been grouped into three categories classified as below-normal (driest third), near-normal (middle third) and above-normal (wettest third). GCM forecast probabilities have been calculated by expressing as a percentage the ensemble members that fall within the upper, middle and lower categories. For example, if 6 ensemble members fall in the above-normal category, 3 in the nearnormal, the other 3 in the below-normal, then the probability forecasts would be expressed as $50 \%$ chance, 
$25 \%$ chance, and another $25 \%$ chance of rainfall occurring in the above-, near-normal and below-normal categories, respectively.

\subsection{The recalibration methods}

Owing to systematic errors inherent in GCM simulations at regional and local scales, adjustments are necessary to improve model predictions at such spatial scales. The statistical transformations, model output statistics (MOS), applied in this study attempt to improve the skill of model predictions using regressionbased techniques. The MOS approach (Wilks, 1995; Landman and Goddard, 2002) statistically links archived records of ECHAM4.5 GCM forecast fields (considered as predictors) and historical rainfall records for the same season. The developed relationships are then used to produce MOS-based forecasts. In this approach, the lead time is incorporated through the GCM forecasts. For example, if DJF GCM forecasts are produced early in October (November) then the lead time is 2 (1) months. The value of the forecasts issued at these lead times, albeit short, is sufficiently high practically and economically, and thus their skill levels are evaluated in this paper.

2.4.1. Principal components regression. This method combines principal component analysis (PCA) and regression. Principal components of the predictor variables (GCM forecast fields) are regressed against the observed rainfall for each region. When dealing with climate data, multiple regression becomes cumbersome owing to the huge datasets involved. In addition, a large number of predictors from neighbouring locations or grid points are inherently highly correlated. The high correlations among the predictor variables result in large error variances in the estimates of the regression coefficients and consequently can result in large forecast errors. Since the principal components are uncorrelated, PCR eliminates the problem of multicollinearity of the predictors (Jackson, 1991). In this study, a regression model was built using a limited number of dominant principal components (as determined from a scree test) of the GCM forecast fields as predictors and standardised rainfall for each region (Figure 1(b)) as the predictand.

2.4.2. Canonical correlation analysis. CCA (Barnett and Preisendorfer, 1987; Jackson, 1991; Wilks, 1995; von Storch and Zwiers, 1999) is similar to PCR by constructing a statistical model based on empirical relationships among variables. CCA, however, identifies pairs of linear combinations among the predictor and predictand variables whose time evolution is optimally correlated. The dominant CCA pair gives the highest canonical correlation, followed by the next pair and so on, subject to the condition that successive time coefficients are orthogonal to the previous ones. It is the procedure of identifying multicomponent predictors and multicomponent predictands exhibiting the strongest link that increases the complexity of CCA relative to PCR, which linearly combines only the predictor variables. In this study, CCA was used to develop a linear statistical model as well as for recalibrating GCM forecasts. The statistical methods used in this research to construct the prediction models have been chosen because of their ability to give guidance with respect to the physical link between the variables.

\subsection{Model selection}

The maximum number of predictor and predictand principal components to be retained for further analysis was determined from the scree test. Using this graphical analogue of a significance test, from the total of $N$ principal components (where $N$ corresponds to the length of each training period), we retained only a subset whose corresponding characteristic roots exceed a certain threshold (determined by the elbow in the graph). In all cases, the retained principal components explained at least $75 \%$ of the original predictor or predictand variance. For further details on scree test, see Jackson (1991) and Wilks (1995). For the PCR models, the optimum number of predictors was further selected on the basis of cross-validated fit over the training period. The cross-validated skill was calculated, starting with only the leading principal component in the model, including the next at each step, and successively adding additional principal components without removing the first few. A similar selection criterion was used for the CCA models. With the CCA modes restrained to be the minimum of the predictor and predictand retained empirical orthogonal function (EOF) 
modes, all the possible combinations were tested and the combination yielding the best cross-validated skill over each training period was selected. The goodness of fit was expressed in terms of correlation between the observed and estimated rainfall.

\subsection{Retroactive forecasting}

In order to get realistic indications of how skilful the models would be in an operational environment, the model performance was assessed over an independent testing period. In this retroactive procedure, the available data were divided into two sets: the 28 years of SST data 1961-1988 (DJF rainfall data 1961/62-1988/89) was initially chosen to be the model fitting or training period for the linear statistical model, and the 3 years 1989-1991 (DJF 1989/90-1991/92) for independent forecasting. After the first 3-year predictions were made, the training period was updated and the model was fitted using the 31 years of SST data 1961-1991 (DJF rainfall data 1961/62-1991/92), and predictions for the next 3 years 1992/93-1994/95 were made. This procedure was repeated using the optimal model for each training period (possibly consisting of different numbers of EOF and CCA mode combinations) until 12 retroactive predictions were made. For the MOS models, the initial training period was set to 21 years (1968/69-1988/89). After making the first retroactive forecast for the 3-year period, 1989/90-1991/92, the model-fitting period was updated at regular steps of 3 years each until the 12 predictions were made.

\subsection{Model validation}

Owing to the uncertainty inherent in long-lead forecasts, the common practice is to present the forecasts probabilistically (Goddard et al., 2001). Accordingly, in this study, the retroactive forecasts were expressed in probabilistic terms. The raw-GCM probabilistic forecasts were derived from the distribution of the ensemble members among the three categories as discussed in Section 2.3. For the statistical models, probabilistic forecasts were obtained from the prediction error variance (Mason and Mimmack, 2002). The probabilistic forecasts were paired with the subsequent observations for each season during the testing period, and probabilistic performance scores were calculated, which were used to quantitatively validate the prediction models. The skill scores used to assess model performance are the ranked probability skill score (RPSS) (Wilks, 1995; Mason and Mimmack, 2002; Toth et al., 2003), and the relative operating characteristics (ROC) areas (Mason and Graham, 1999; Mason, 2003). These skill scores measure different aspects of forecast performance, but do not give a complete picture (Wilks, 1995). The RPSS gives an overall (all categories) measure of the skill of probabilistic forecasts, while the ROC areas indicate the ability of the forecast system to discriminate between individual categories.

The most commonly used reference strategy for calculating the RPSS is climatology, in which the prior probability of the event being forecast is issued perpetually. In the case of the three equiprobable categories, climatological forecasts assign $33.3 \%$ of the probability to each category. Climatology generates a negatively biased skill score, and so random guessing, where the outcome is independent of the forecast, is an unbiased alternative strategy, but the resultant score is not strictly proper (Mason, 2004). In this research, skill scores for the competing forecast systems have been calculated with reference to climatology.

The ROC score gives estimates of forecast skill for individual categories. Hence these scores were calculated for each of the three categories separately. However, it is only forecasts of extreme categories that are of concern to human, agriculture, and economic welfare of society. Accordingly, only results for the above- and below-normal categories are presented in this paper. In any case, the skill scores for predicting the near-normal category are very low.

\subsection{Significance tests}

The statistical significance of the differences between skill scores for competing forecast schemes is determined through a Monte Carlo or resampling technique (Wilks, 1995). In this non-parametric approach to significance testing, 1000 artificial batches of the same size at the actual data are constructed using timeshuffled versions of the original precipitation data. The test statistics of interest (i.e. RPSS and ROC scores) 
are then computed for each artificial batch, and differences between the forecast schemes' scores calculated. The differences between the scores are then sorted and constitute a sampling distribution against which the differences computed from the original data are compared. For a 1000 values, the 950th ranked value corresponds to the $95 \%$ confidence level threshold.

\section{RESULTS}

\subsection{Optimal model selection}

The GCM hindcast mode forecast fields considered as candidate predictors for the MOS models were the geopotential heights $(1000,850,700$, and $500 \mathrm{hPa})$, total precipitation, and total cloud cover. The difference in cross-validated correlation over the four model-fitting periods using each GCM output field as a predictor in the MOS models is noticeable, albeit small. Among all the potential predictors, the 850-hPa geopotential height proved the best predictor of austral summer rainfall over southern Africa, consistent with findings by Landman and Goddard (2002). The 700-hPa geopotential height field is the second best predictor, followed by $1000 \mathrm{hPa}$, and then $500 \mathrm{hPa}$. The GCM total precipitation and cloud cover produced the least overall skill. However, the rerandomisation tests rendered the differences between the cross-validated skill scores obtained using each forecast field as a predictor in the MOS models statistically insignificant at the $95 \%$ confidence level. For example, at 1- (2-) month lead time, the 21-year training period is associated with a $95 \%$ confidence level threshold of cross-validated correlation difference between the 850 -hPa geopotential height and total cloud cover of $0.1031(0.1816)$, the 24-year period with $0.1391(0.2031)$, the 27-year period with 0.1087 (0.2308) and the 30-year period with 0.1319 (0.2517). The actual differences in cross-validated correlation do not exceed these threshold values.

The $850-\mathrm{hPa}$ geopotential height field is acceptably well simulated by the GCM and hence appropriate as a predictor of regional rainfall. Much of SADC is more than $1000 \mathrm{~m}$ above mean sea level, thus the $850 \mathrm{hPa}$ should provide a good representation of low-level synoptic circulation, which is one of the dominant factors determining regional climate variability over the interior plateau.

The selected optimal combination of predictor and predictand EOF modes retained in the CCA analysis, and the number of CCA pairs used in each statistical model are presented in Table I. The training periods (defined according to the length of the predictor data), target periods and their corresponding lead times are also shown. Minor discrepancies notwithstanding, there is some consistency in selecting the number of SST and rainfall EOFs, and CCA modes making up the optimal linear statistical model for each training period. There are only minor differences in the combination making up the optimal model for the 1961-1997 training period. This consistency makes sense given that the training periods have many years in common. For the CCA-MOS models, however, the number of the EOFs and CCA modes change each time the training period is updated.

The optimum number of principal components (PCs) of the predictor field used in the PCR model is shown in Table II. The number of skilful predictor PCs ranges from 2 to 5 . In each case, about $58 \%$ of the total variance is explained by the first two leading modes. In this study, the PCs were entered into the model according to the magnitudes of their associated characteristic roots. Whereas the first mode in each case appeared the most important predictor, the selection criteria used was designed to ensure that additional PCs would only be allowed into the model if they make a contribution to the forecast skill.

\subsection{Performance of the models}

The RPSS for the retroactive forecasts calculated at 2- and 1-month lead times are shown in Figure 2. The baseline skill represented by raw-GCM ensemble forecasts and the CCA linear statistical model are also plotted to give a visual comparison of the model scores. The graphs show the RPSS at 2- and 1-month lead times calculated relative to a strategy of perpetually forecasting the climatological probabilities of each of the three categories. 
Table I. The different combinations of the number of predictor (SST) and predictand (PRCP) EOFs, and the number of CCA modes used in the CCA linear statistical (top-part) and the CCA-MOS (bottom-part) models developed for each retroactive period

\begin{tabular}{|c|c|c|c|c|c|c|}
\hline \multirow[b]{2}{*}{$\begin{array}{l}\text { Target } \\
\text { season }\end{array}$} & \multirow[b]{2}{*}{$\begin{array}{l}\text { Training } \\
\text { period }\end{array}$} & \multirow[b]{2}{*}{$\begin{array}{l}\text { Lead time } \\
\text { (months) }\end{array}$} & \multicolumn{2}{|c|}{ Number of EOF modes } & \multirow[b]{2}{*}{ CCAs } & \multirow[b]{2}{*}{ Correlation } \\
\hline & & & SST & PRCP & & \\
\hline \multirow[t]{17}{*}{ DJF } & $1961-1988$ & 2 & 8 & 5 & 5 & -0.049 \\
\hline & $1961-1991$ & 2 & 8 & 5 & 5 & -0.013 \\
\hline & $1961-1994$ & 2 & 8 & 6 & 5 & 0.019 \\
\hline & $1961-1997$ & 2 & 5 & 4 & 3 & 0.022 \\
\hline & $1961-1988$ & 1 & 7 & 5 & 5 & 0.009 \\
\hline & $1961-1991$ & 1 & 8 & 5 & 5 & 0.015 \\
\hline & $1961-1994$ & 1 & 6 & 5 & 5 & 0.041 \\
\hline & $1961-1997$ & 1 & 6 & 3 & 3 & 0.061 \\
\hline & & & $850-\mathrm{hPa}$ & PRCP & CCAs & \\
\hline & 1968/69-1988/89 & 2 & 5 & 3 & 2 & 0.047 \\
\hline & $1968 / 69-1991 / 92$ & 2 & 5 & 6 & 3 & 0.091 \\
\hline & $1968 / 69-1994 / 95$ & 2 & 3 & 4 & 1 & 0.117 \\
\hline & $1968 / 69-1997 / 98$ & 2 & 3 & 1 & 1 & 0.112 \\
\hline & $1968 / 69-1988 / 89$ & 1 & 5 & 3 & 2 & 0.267 \\
\hline & $1968 / 69-1991 / 92$ & 1 & 5 & 5 & 2 & 0.295 \\
\hline & 1968/69-1994/95 & 1 & 4 & 1 & 1 & 0.257 \\
\hline & $1968 / 69-1997 / 98$ & 1 & 4 & 1 & 1 & 0.251 \\
\hline
\end{tabular}

Table II. The optimal number of dominant GCM 850-hPa geopotential heights used in the PCR recalibration models developed for each retroactive period

\begin{tabular}{lcccc}
\hline \multirow{2}{*}{ Target season } & Training period & Lead time (months) & $\begin{array}{c}\text { Number of EOF modes } \\
850 \mathrm{hPa}\end{array}$ & Correlation \\
\hline DJF & $1968 / 69-1988 / 89$ & 2 & 4 & 0.011 \\
& $1968 / 69-1991 / 92$ & 2 & 5 & 0.035 \\
$1968 / 69-1994 / 95$ & 2 & 5 & 0.024 \\
$1968 / 69-1997 / 98$ & 2 & 5 & 0.031 \\
$1968 / 69-1988 / 89$ & 1 & 4 & 0.201 \\
$1968 / 69-1991 / 92$ & 1 & 5 & 0.195 \\
$1968 / 69-1994 / 95$ & 1 & 5 & 0.154 \\
& $1968 / 69-1997 / 98$ & 1 & 2 & 0.180 \\
\hline
\end{tabular}

It is evident from the graphs that the raw-GCM ensembles perform least well. At both lead times, over most regions, the RPSS values for the raw-GCM forecasts are low and negative suggesting that the probabilities calculated from the distribution of the GCM ensembles are worse than the use of climatological probabilities (33\% for each of the three categories). Positive but still low RPSS values are found over central Mozambique, southern Zambia, and northern Botswana and Zimbabwe at 1-month lead time. In a majority of cases, the RPSS values for the MOS models are higher than those for the raw GCM. The differences in the RPSS for the MOS models and the raw-GCM ensembles are statistically significant at the $95 \%$ confidence level at both lead times.

There are some noticeable improvements in the skills of the recalibration methods compared to those of the CCA linear model, but in a few cases, the recalibration models are slightly outperformed by 
(a) RPSS at two-months lead-time

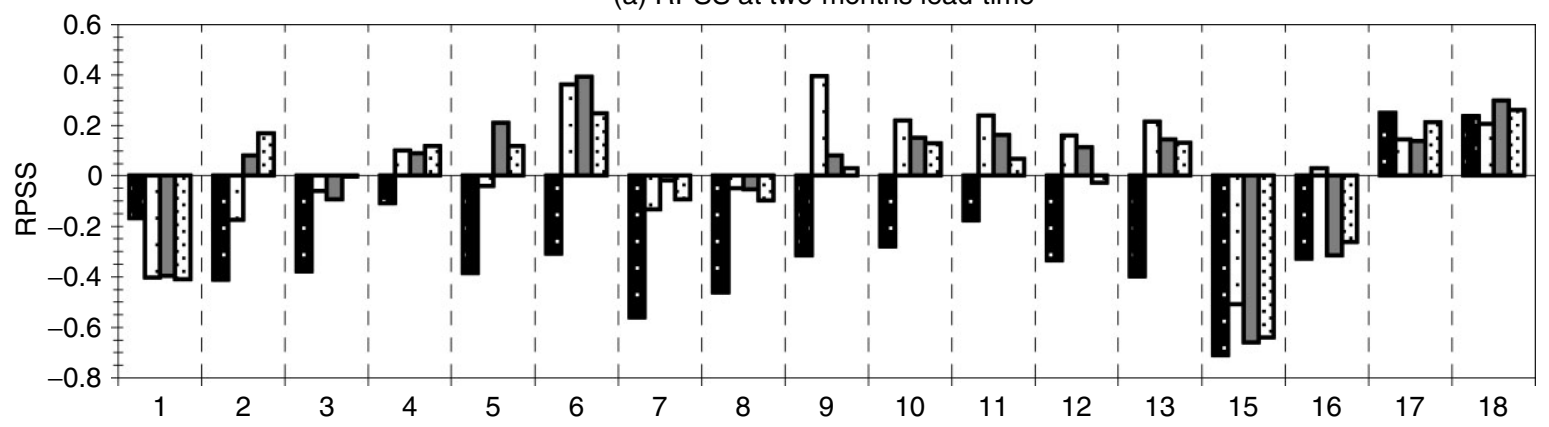

(b) RPSS at one-month lead-time

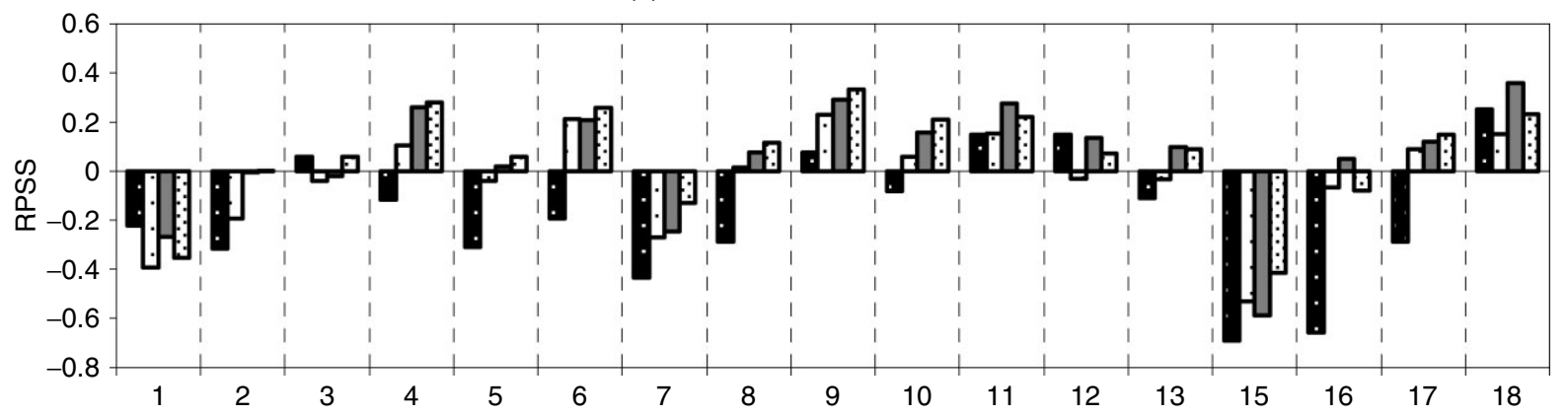

Figure 2. Ranked probability skill scores for retroactive forecasts at (a) 2- and, (b) 1-month lead time for the 18 rainfall regions (for region locations, see Figure 1(b)). The skill scores have been based on climatology as the reference strategy. The black bars represent the scores for the raw-GCM ensembles, the white dotted bars (second series) for the CCA linear statistical, the dark grey bars (third series) scores for the PCR-MOS model, and the light grey bars with several dots (fourth series) represent scores for the CCA-MOS model. No data were available over eastern Angola (region 14)

the simple statistical model, and their score differences are statistically insignificant at $95 \%$ confidence levels. In general, the SST-rainfall model was more successful at capturing the anomalously dry periods experienced over the southern parts of SADC during the early 1990s but not equally skilful during the latter part of the validation period. This is to be expected given that the recurrent droughts experienced over the southern areas during the early 1990s were partially in response to anomalous SST forcing mainly from the Pacific Ocean. Whenever the ocean appears to drive atmospheric anomalies, any model in which ocean surface parameters (such as SSTs) are the sole predictors should predict the atmospheric response acceptably well, particularly if the relationships determined during the training period remain stable during the model validation or forecast period. On the other hand, the MOS models appear to perform consistently well during each retroactive period, giving higher probabilities to the category that occurred even during years characterised by the absence of strong anomalous SSTs over the major ocean basins.

Averaged over the whole region, the RPSS for each model at the different lead times (2-; 1-month) are: GCM (-0.28;-0.18), Linear (0.04;-0.01), PCR-MOS (0.02; 0.05), and CCA-MOS (0.002; 0.06). It can be seen that overall, the skill of the raw GCM and the MOS models improve at the shortest lead time (1-month). Whereas the PCR-MOS model performs slightly better than the CCA-MOS model on several occasions, neither type of recalibration technique shows a clear superiority over the other. The differences in their skill scores at both lead times are not statistically significant. This result is not surprising; both models are regression-based, and are closely related by developing a linear relationship between archived GCM forecast fields and regional rainfall. Yu et al. (1997) compared the performance of CCA and PCR in predicting seasonal rainfall variations in the United States affiliated Pacific islands from SSTs in the Pacific Ocean. Consistent with our findings, they also noted comparable levels of skill from the two statistical methods. 
(a) ROC scores for below-normal category at two-months lead-time

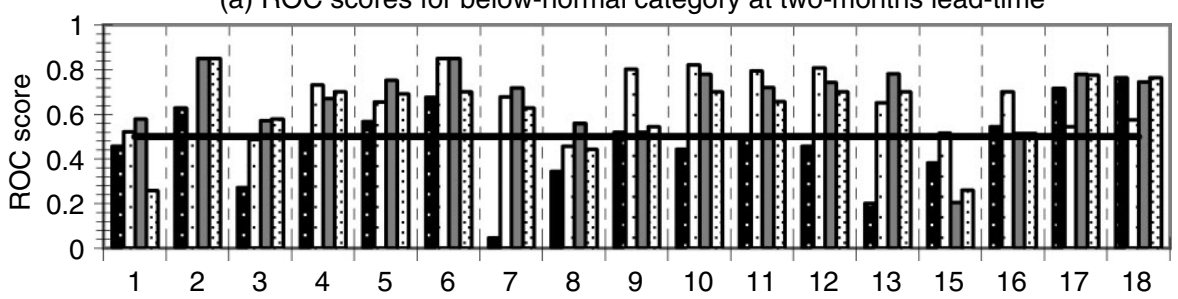

(b) ROC scores for below-normal category at a one-month lead-time

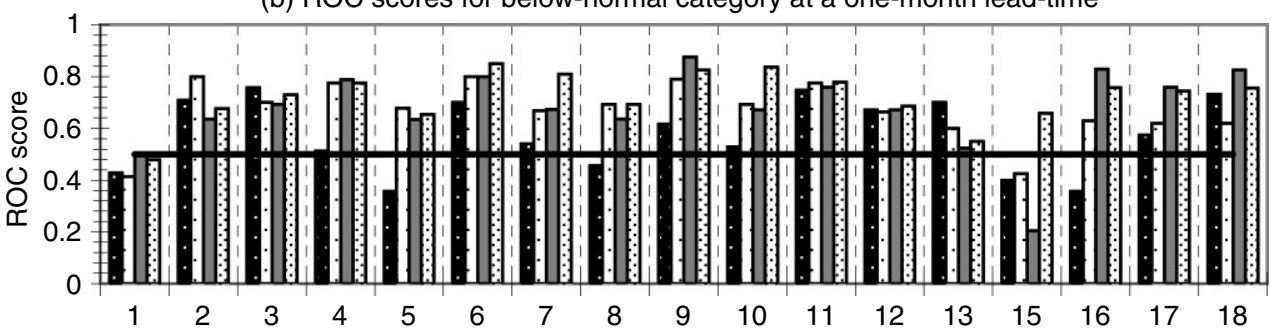

(c) ROC scores for above-normal category at two-months lead-time

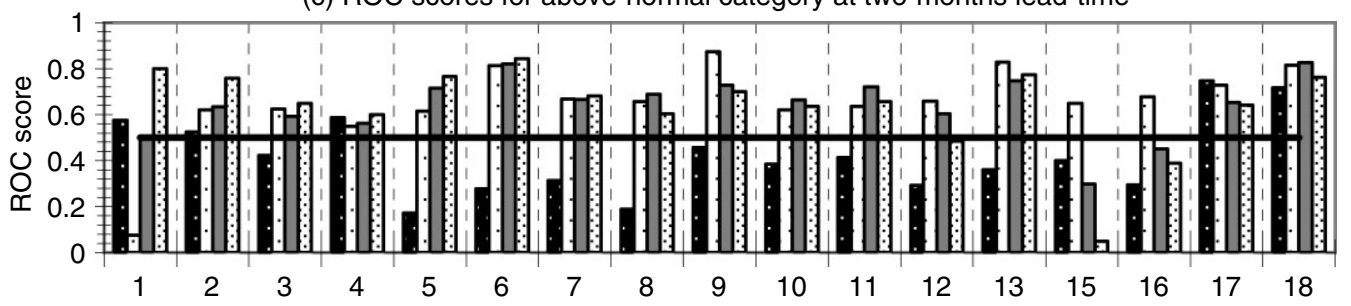

(d) ROC scores for above-normal category at a one-month lead-time

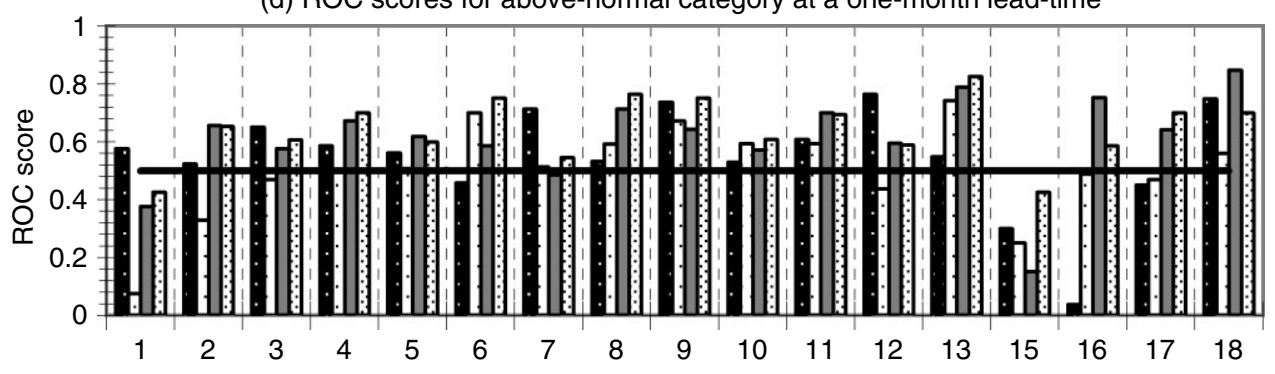

Figure 3. ROC areas for below- and above-normal categories, and for the 18 rainfall regions (as shown in Figure 1(b)). Scores are for: raw-GCM ensembles (black bars), the white dotted scores (second series) for the CCA linear statistical, the grey bars (third series) scores for the PCR-MOS model, and white bars with several dots (fourth series) represent scores for the CCA-MOS model. The 0.5 line is shown

The ROC scores (area under the ROC curve) for retroactive forecasts of extreme rainfall categories (aboveand below-normal) in each region, are shown in Figure 3. For skilful forecast systems ROC scores exceed 0.5 (shown by the line in the figure), consistent with a ROC curve lying above the diagonal where hit-rates are higher than false-alarm rates. ROC scores equal to 0.5 imply little or no useful information while those less than 0.5 are indicative of negative forecast skill. For details on ROC scores and their interpretation, see Mason and Graham (1999), and Mason (2003). The ROC scores echoes RPSS findings that raw output from the GCM is inferior to its competitors. However, the ROC scores show promising results in that the GCM has more positive skills, particularly at 1-month lead time. This result suggests that the numerical model is capable of predicting extreme categories with some skill. Despite the fact that the GCM does a better job in predicting extremes, the MOS models continue to dominate the numerical model as shown by the statistically 
significant differences in their ROC scores at 2-months lead time. Consistent with the results revealed by the RPSS, the ROC scores show that DJF rainfall over the southwestern Cape, and part of northern Zambia and western Tanzania (regions 1 and 15) are the least predictable, overall. The scores indicate that the CCA linear statistical model and the recalibration models appear to be able to predict both below- and above-normal DJF rainfall over much of the SADC region with comparable levels of skill. Minor (and statistically insignificant) differences in the scores of the PCR- and CCA-MOS notwithstanding, there are still no clear indications of superiority of one model over the other.

\section{POSSIBLE SOURCES OF PREDICTIVE SKILL}

In this section, the statistical link between regional rainfall and large-scale features of ocean variability, and the GCM large-scale circulation are investigated. The CCA spatial patterns and their corresponding temporal scores are used to diagnose possible mechanisms underlying the predictability of regional rainfall from largescale features of the climate system. For brevity, only the first two pairs of canonical correlation patterns (exhibiting the highest canonical correlations, hence considered to be contributing the most to the forecast skill) are presented in each case.

\subsection{SST-rainfall link}

To draw inferences about the nature of ocean-atmosphere interaction responsible for the predictability of southern Africa rainfall from near-global SSTs, the temporal variability of the canonical coefficients and the spatial patterns are investigated. In a broad sense, the predictor and predictand-loading patterns at 1- and 2-month lead times are similar. This similarity is to be expected from slowly evolving boundary-forcing features such as SSTs. For this reason, only the patterns obtained with the SSTs leading rainfall by 2 months are presented here. The link between SSTs and regional rainfall are discussed using only the first two CCA pairs. The spatial patterns and the associated time scores are shown in Figure 4.

CCA mode 1 describes the most significant source of predictability of DJF rainfall variability from SSTs. The SST series for this mode explains $29 \%$ of the total variance. The correlation coefficient between the two canonical component time series is 0.64 (Figure 4(c)). The SST predictor loading patterns (Figure 4(a)) for this mode suggest that most forecast skill comes from the tropical Pacific SST forcing. The spatial pattern includes strong negative loadings over much of the eastern tropical Pacific Ocean. Strong predictor loadings in the equatorial Pacific Ocean suggest the presence of the El-Niño/Southern Oscillation (ENSO) in the relationship with southern Africa DJF rainfall. The correlation between the SST 1961-1997 time series for this mode and the Southern Oscillation Index is 0.41 (statistical significance at the 95\% level). The DJF predictand-loading pattern (Figure 4(b)) shows mainly positive values over much of the southern regions (regions 2-12) and negative values over the northern part (regions 13-18). Inspection of the predictor and predictand spatial patterns reveal that warmer (cooler) SSTs have often occurred in the equatorial Pacific Ocean from July-September during years characterised by below (above) average DJF rainfall over homogeneous rainfall zones south of about $15^{\circ} \mathrm{S}$ and vice versa over the northern part. This result concurs with previous findings (e.g. Ropelewski and Halpert, 1987).

CCA mode 2 presents the second most important source of DJF rainfall predictability. The predictor pattern for this mode explains $21 \%$ of the total SST variance. The canonical correlation is 0.49 . The canonical time series (Figure 4(f)) suggest that this mode captures the influence of long-term trends or interdecadal mode of SST variability on DJF precipitation. The SST loading pattern (Figure 4(d)) shows broad positive loadings over the Pacific Ocean, with strongest loadings over the northern Pacific. Positive loadings are also found south of the African continent. Longer-period (interdecadal) oscillations in the major oceans, notably the Pacific have been widely discussed (e.g. Gershunov and Barnett, 2003). From the 1960s to the early 1970s, there seems to have been a cooling trend in SSTs over much of the northern Pacific Ocean, followed by a reverse pattern from the mid-1970s onward. The predictand-loading pattern (Figure 4(e)) suggests that mode 2 is associated with a dipole structure of DJF rainfall anomalies between the northern and southern parts of 
(a) SST JAS mode 1

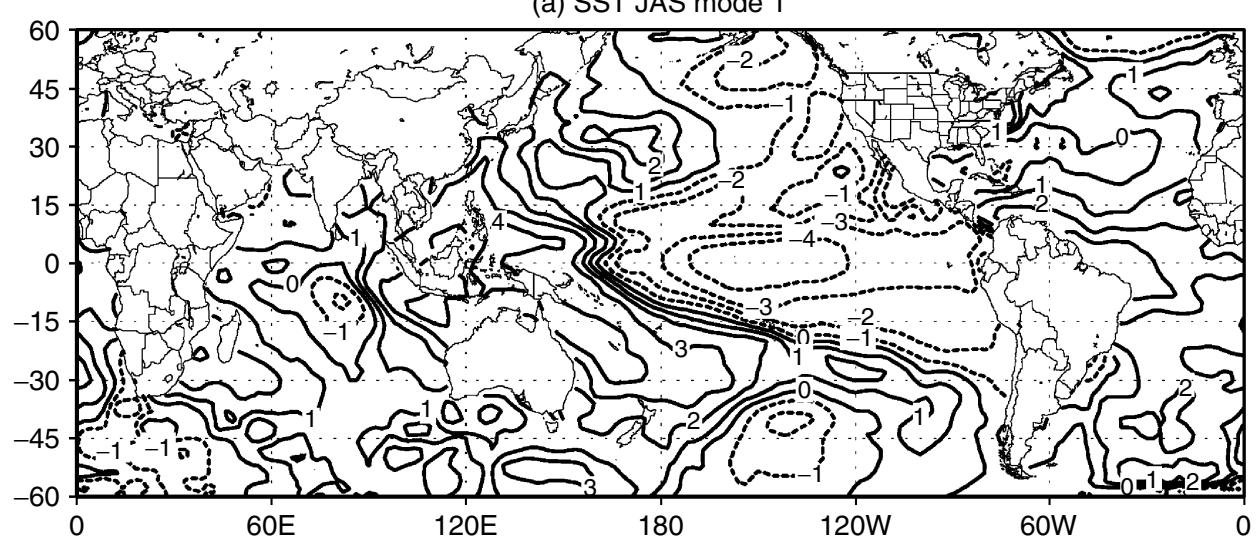

(b) DJF PRCP mode 1

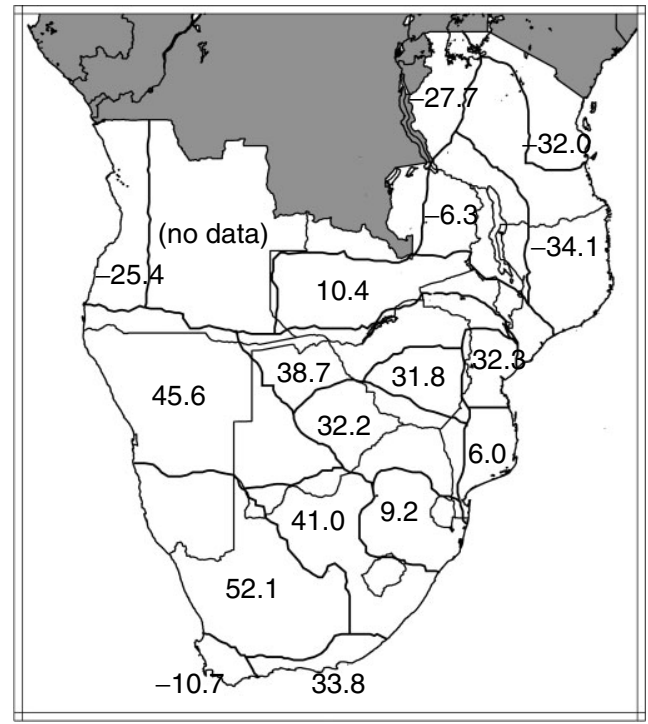

(c) Temporal scores mode 1

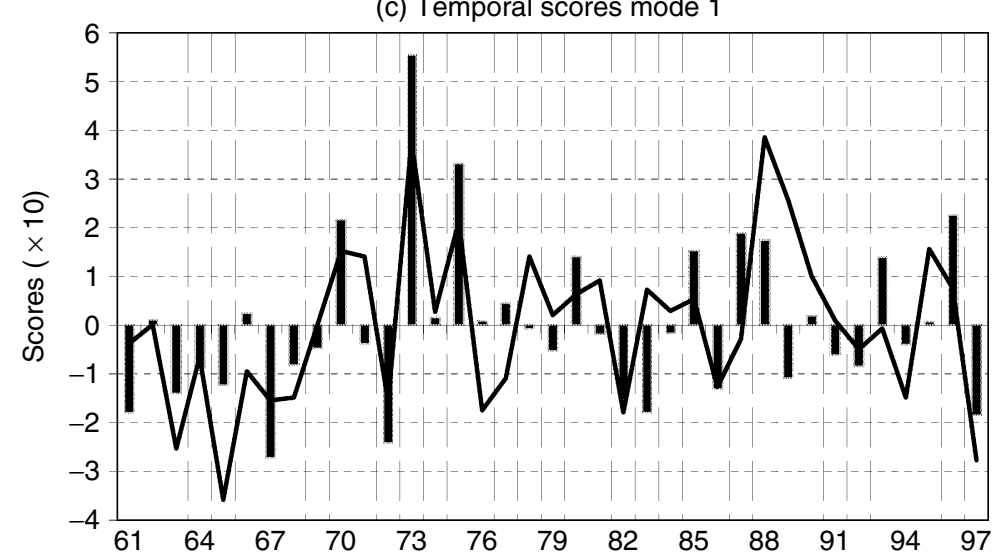

Figure 4. Canonical correlation maps and temporal scores for the first two CCA pairs for JAS SSTs and DJF rainfall. First (a) predictor and (b) predictand mode spatial loadings $(\times 10)$. (d) and (e) show similar patterns but for the second mode. The corresponding 1961-1997 temporal scores for SSTs (dark line) and precipitation (bars) are shown in (c) and (f) for mode 1 and mode 2 respectively 
(d) SST JAS mode 2

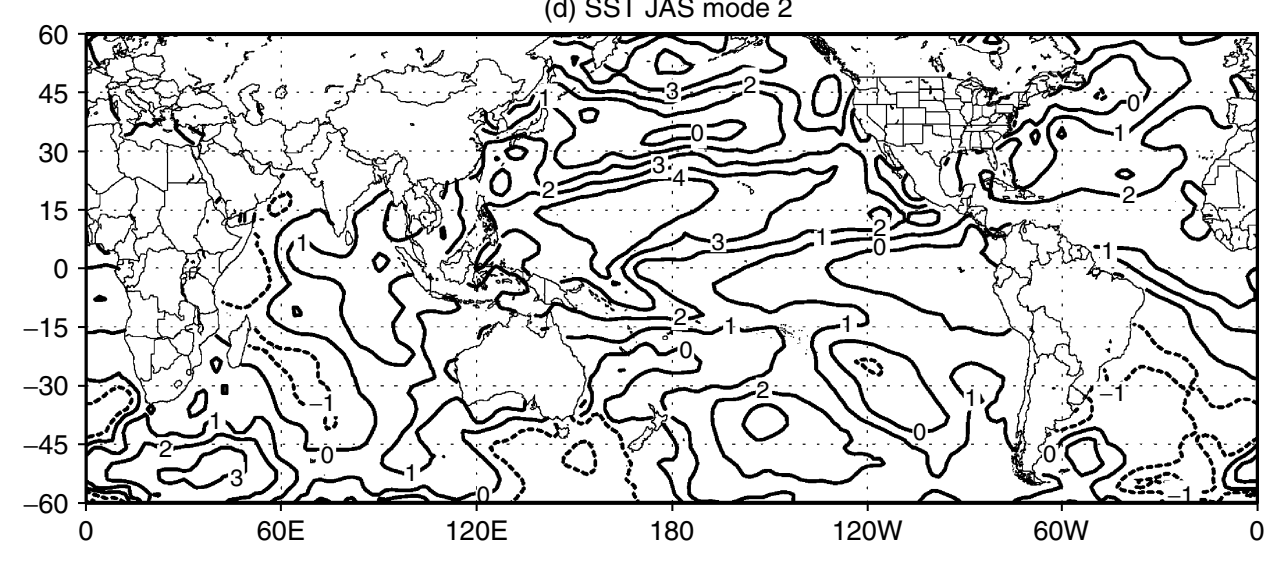

(e) DJF PRCP mode 2

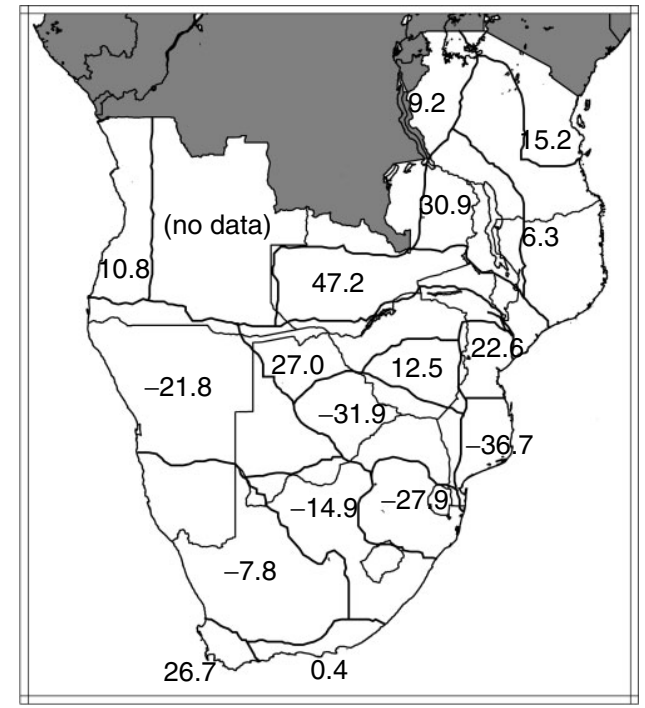

(f) Temporal scores mode 2

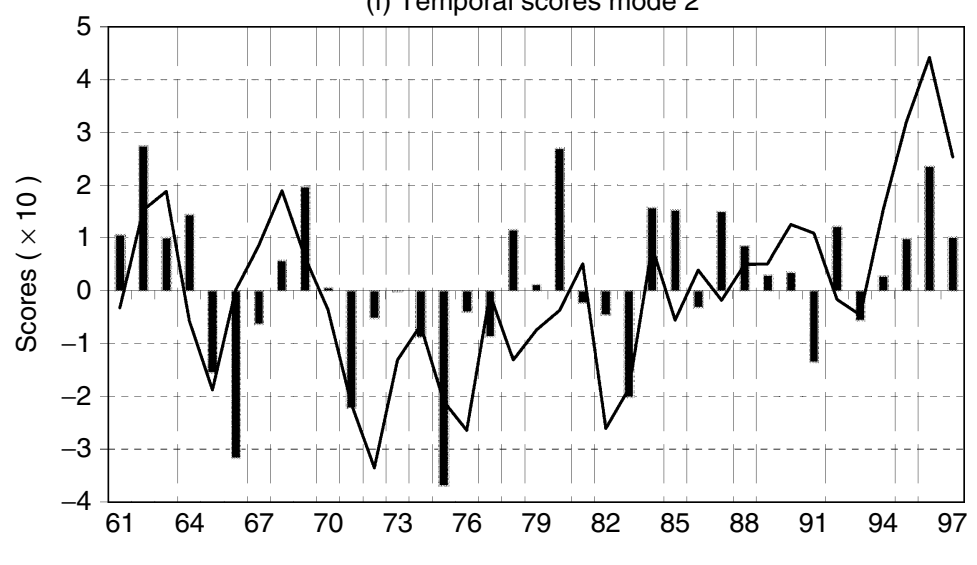

Figure 4. (Continued) 
SADC. That is northern Pacific Ocean warming from the mid-1970s has been associated with diminishing DJF rainfall over the southern sector (an exception being the southern tip of South Africa), and vice versa to the north.

\subsection{GCM circulation field}

The statistical link between the GCM 850-hPa geopotential height and DJF rainfall over SADC is again discussed based on the first two canonical patterns. There are some differences in canonical patterns at the two different lead times used in this research. However, in the interest of brevity only the patterns for MOS forecasts produced at a 1-month lead, which has been shown to be more skilful, are presented.

The first CCA pair, shown in Figure 5(a), (b) associates higher (lower) geopotential heights over much of the subcontinent and the bordering southeastern Atlantic Ocean with negative (positive) DJF rainfall anomalies over much of central and southern SADC. The $850-\mathrm{hPa}$ geopotential height pattern for this mode explains $37 \%$ of the total variance. The correlation between the respective canonical vectors is 0.78 (Figure 5(c)). This pattern represents a meaningful physical relationship: if the low-level pressure (as represented by the 850-hPa geopotential height over high-elevation inland areas) is high (low), atmospheric stability (instability) prevails and vertical motion is suppressed (enhanced) resulting in diminished (enhanced) rainfall over the region.

The second canonical correlation pattern is shown in Figure 5(d), (e). The predictor pattern for this mode explains $25 \%$ of the total variance. The correlation between the canonical vectors is 0.74 (Figure 5(f)). High (low) geopotential height over the southwestern Indian Ocean, southeast of Madagascar is associated with positive (negative) DJF rainfall anomalies over much of the southern sector, and vice versa over the north and southern tip of South Africa. This pattern is quite reasonable physically in view of the fact that the southwestern Indian Ocean is a major source of moisture for precipitation formation over southeastern SADC. High (low) 850-hPa geopotential heights connote an intense (weak) southwestern Indian Ocean (Mascarene) anticyclone, which may persist even up to the 700-hPa level, resulting in enhanced (weakened) advection of moisture inland. Sufficient (insufficient) moisture advection inland is consistent with enhanced (diminished) rainfall over southeastern SADC.

\section{DISCUSSION AND CONCLUSIONS}

Disparate climate regimes exist within SADC resulting from different regional forcings. On the basis of 255 rainfall stations across the region, 18 homogeneous climate zones have been identified from cluster analysis. These zones are believed to experience similar characteristics in terms of their seasonal to interannual rainfall variability. Of the 18 zones, some receive their maximum amount of rainfall outside the austral summer rainfall months. These include the austral winter rainfall region along the southwestern coast of South Africa (region 1) and the bimodal rainfall regime of western Tanzania (part of region 15). The apparent lack of skill in predicting DJF rainfall over these zones is therefore not a major concern.

A linear statistical model linking near-global SSTs to regional rainfall has been developed and its skill assessed over a 12-year retroactive period 1989/90-2000/01. This model together with the raw-GCM ensembles has been used as a baseline reference against which the skill of the MOS models can be judged. The model demonstrates usable skill in predicting summer rainfall and has proved to be a strong competitor to state-of-the-art forecast techniques.

The diagnostic features of CCA reveal that the Pacific Ocean is a very important contributor to the prediction skill during the peak austral summer months. This is indicative of the influence of ENSO on southern African rainfall. The CCA predictand patterns exhibit a structure with loadings of opposite signs over the region. This demonstrates that rainfall response to Pacific SST forcing is not uniform across the SADC region, consistent with previous findings (e.g. Ropelewski and Halpert, 1987; Nicholson and Kim, 1997). The patterns show that in those years when southern SADC (south of about $15^{\circ} \mathrm{S}$ ) experience anomalously dry (wet) conditions partly in response to Pacific SST forcing, anomalously wet (dry) conditions are often observed over the north. The ocean basins bordering southern Africa also contribute to the predictability of rainfall during the peak 
(a) $850 \mathrm{hPa}$ DJF mode 1

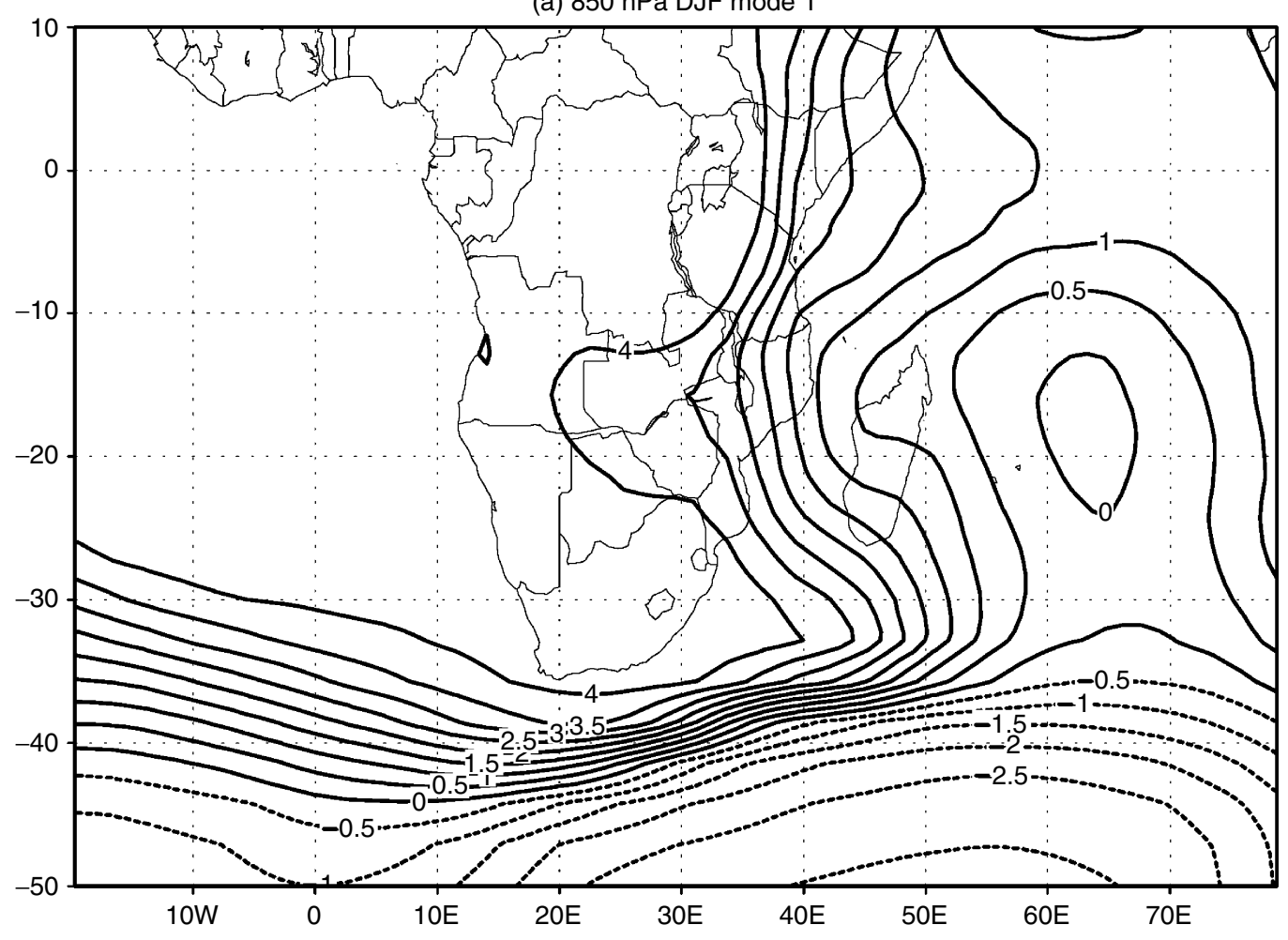

(b) DJF PRCP mode 1

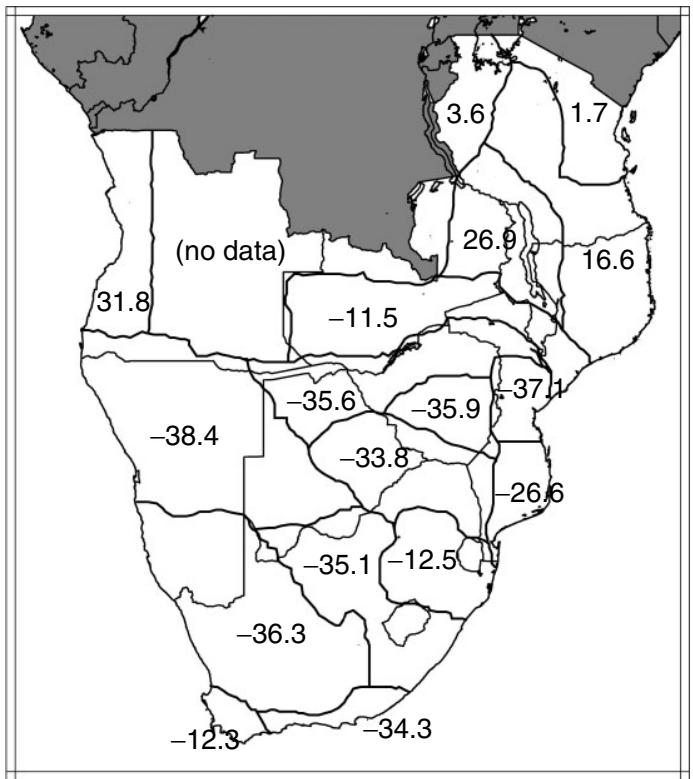

Figure 5. Canonical correlation maps and temporal scores for the first two CCA pairs for the DJF GCM 850-hPa geopotential height and DJF rainfall. First (a) predictor and (b) predictand mode loading patterns $(\times 10)$. (d) and (e) show similar patterns but for the second mode. The corresponding 1961-1991 temporal scores for the GCM 850-hPa geopotential heights (dark line) and precipitation (bars) are shown in (c) and (f) for mode 1 and mode 2 respectively 
(c) Temporal scores mode 1

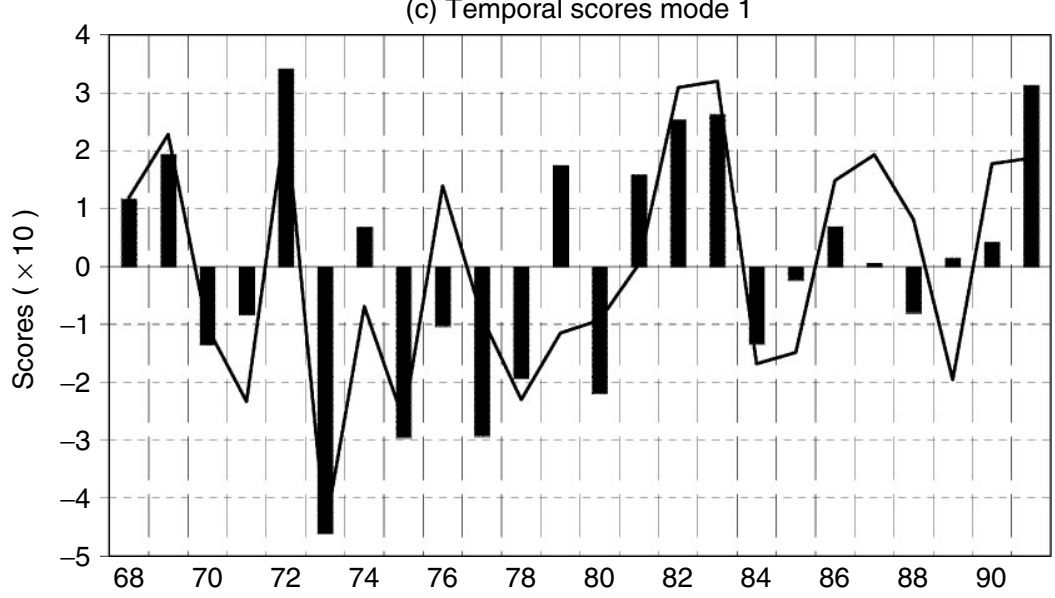

(d) $850 \mathrm{hPa}$ DJF mode 2

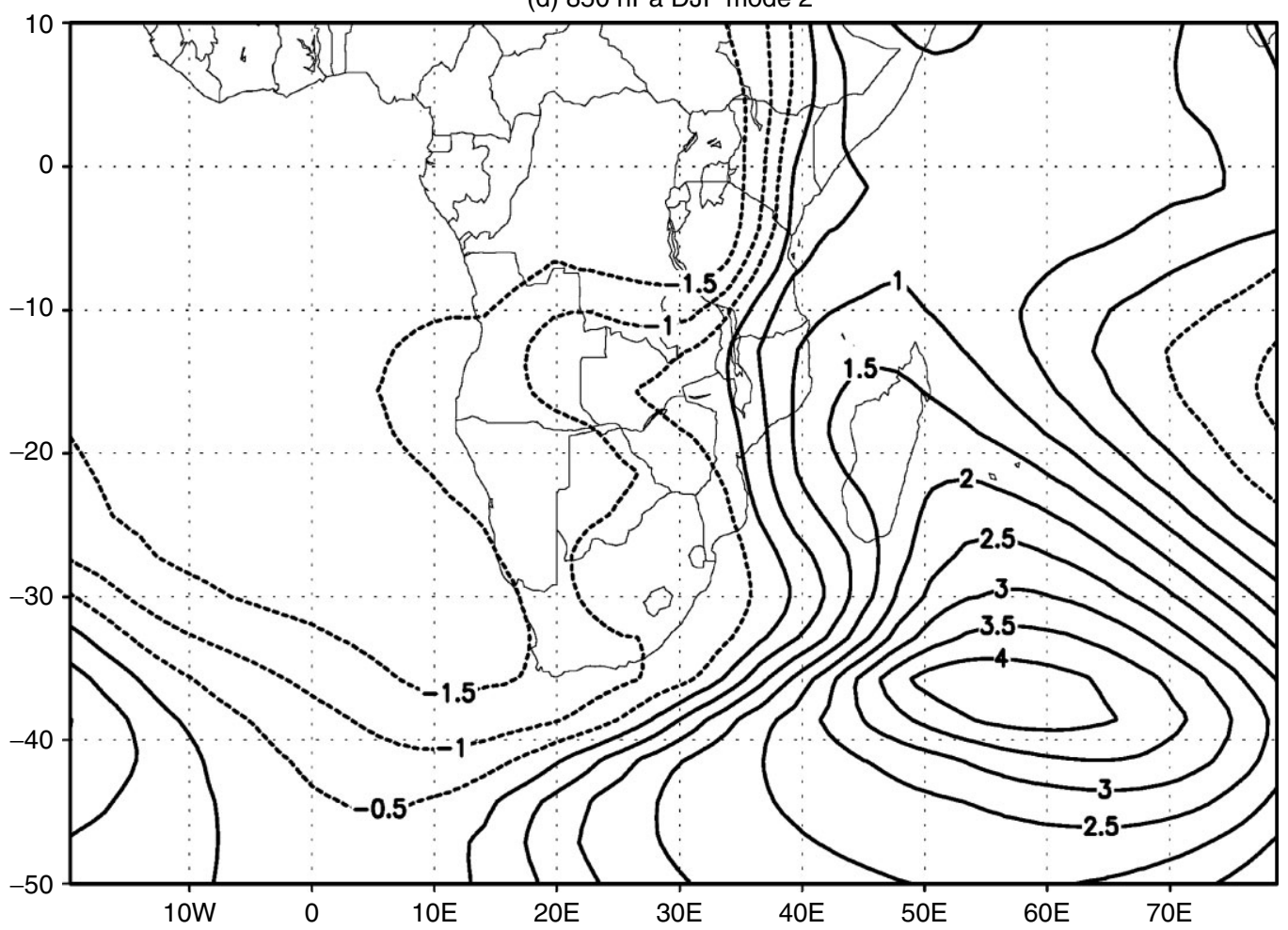

Figure 5. (Continued)

season, and in some cases modulate the effect of anomalous Pacific SST forcing. Noting that ENSO represents a dominant mode in the ocean-atmosphere coupled system, the inability of some GCMs (e.g. ECMWF S1 and S2; van Oldenborgh et al. (2005)) to capture the ENSO teleconnections over southern Africa is discouraging.

Over and above the influence of ENSO, rainfall anomalies are found to be influenced by trends and longerterm or interdecadal variations in the global SSTs. This mode reveals that the global SST field has undergone significant changes during the past few decades, and our results suggest that the SADC austral summer rainfall pattern is sensitive to lower-frequency modes of variability in the global oceans. Again, the rainfall response to interdecadal variations in SST patterns is not uniform across the region. To the south of the region (an 
(e) DJF PRCP mode 2

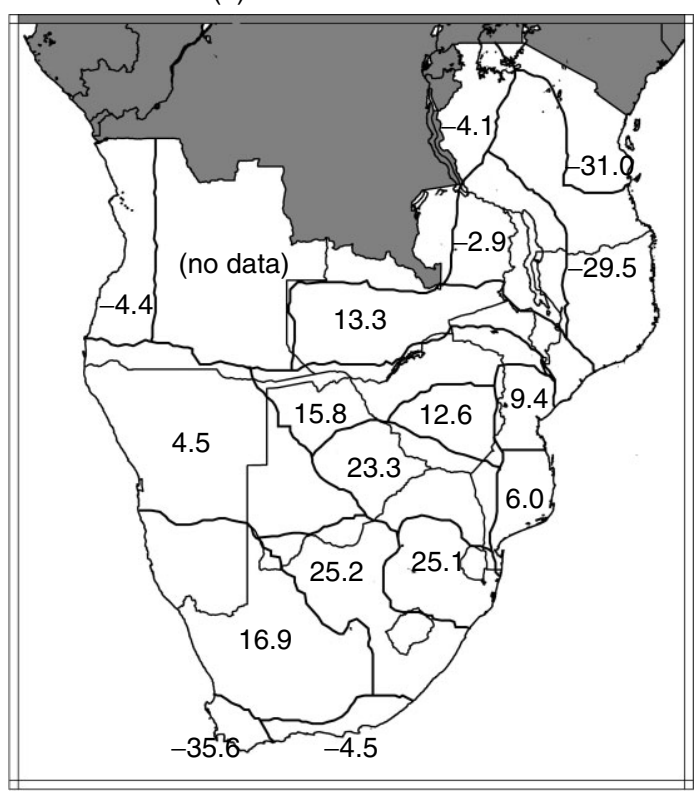

(f) Temporal scores mode 2

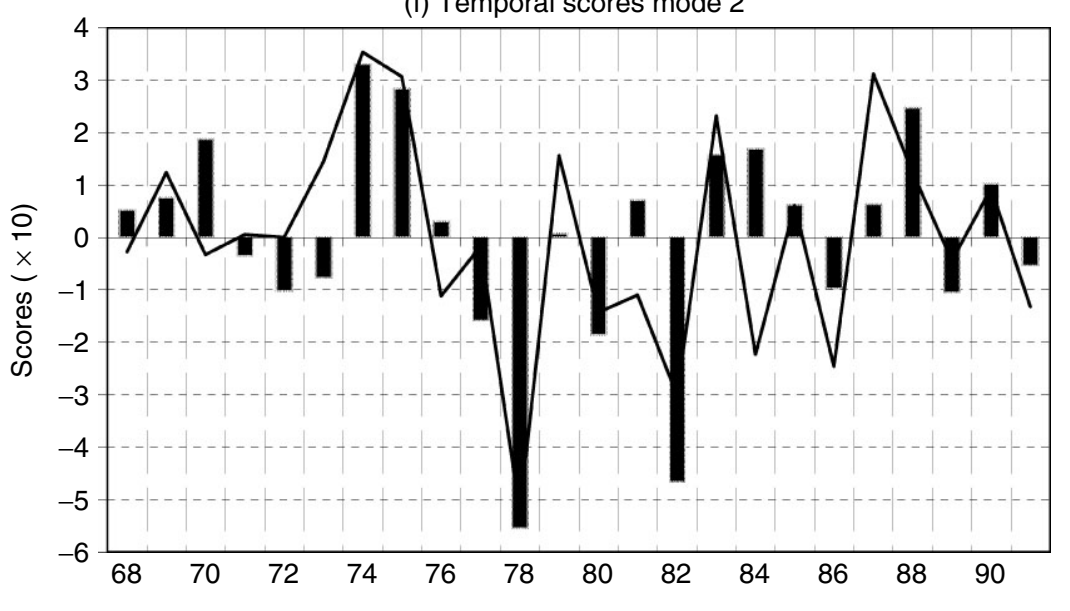

Figure 5. (Continued)

exception being the southern tip of South Africa), there has been a shift in the mean rainfall towards dryer values since the late 1970 s.

The raw-GCM ensembles have been proved to be the poorest performers at the smaller spatial scales considered here. This is a ubiquitous problem with low-resolution numerical models such as the ECHAM4.5 T42 GCM used here. Even the simpler linear statistical model outscores this dynamical model, which attempts to accurately represent the physics of the climate system. The reasons for the poor performance of the GCM are probably related partly to coarse model resolution resulting in inadequate representation of the earth's structure in the model, and partly to parameterisation schemes used. At the shortest lead time (1-month), the model skill improves but it still performs poorer than the other models. This suggests that information from the GCM at shorter lead times may still be useful if considered with care.

The ECHAM4.5 GCM output/rainfall relationship is in agreement with Landman and Goddard (2002), in which the 850-hPa geopotential height from an earlier version of the same model (ECHAM3.6) was found to be the most skilful predictor of southern Africa austral summer rainfall. However, the statistically insignificant 
differences in cross-validated skill over each training period using each of the GCM forecast fields suggest that the other fields (see Section 3.1) can still be useful predictors in the MOS models. Much of inland SADC has an altitude greater than $1000 \mathrm{~m}$ above mean sea level. The $850-\mathrm{hPa}$ geopotential therefore gives a good representation of the low-level circulation. Empirical relationships between archived records of the GCM 850$\mathrm{hPa}$ geopotential height have been derived using two regression-based techniques. These MOS models are based on PCR and CCA. The inherent advantage of applying these methods in recalibrating GCM simulated fields is that complex non-linear relationships among climate variables, which they cannot accommodate alone, are incorporated in the GCM simulations. This study has shown that recalibrating low-resolution model forecasts offers an improvement to the model skill and provides more useful climate information at relatively small spatial scales. PCR-MOS and CCA-MOS offer comparable levels of forecast skill, despite the fact that CCA is a more sophisticated linear model than PCR.

The models have been tested over a 12-year retroactive period and their skill evaluated quantitatively. On average, the skills obtained for the DJF season are high indicating a potential for skilful operational seasonal forecasts using the methods tested in this study. During the early 1990s, ENSO-related anomalously dry conditions persisted and have been skilfully predicted by the models. The models' ability to predict anomalous rainfall events is encouraging. Of the reported natural disasters within the SADC region, those related to weather/climate dominated. Droughts have occurred recurrently over the region resulting in severe deficiencies in soil moisture and hence failure in agricultural production. The severe effects of droughts are often prolonged. Advance guidance regarding a likely shift in the seasonal rainfall pattern, such as expected rainfall suppression in the region would therefore be very important for planning purposes in the agricultural industry and for other socio-economic activities.

The 12-year testing period used here may not be long enough to draw robust conclusions about the operational value of the model forecasts and the stability of the skill in future. The overall objective of this study was to assess if more complicated climate forecasting methods clearly outperform simpler ones. It has long been noted that coarse-resolution numerical models perform poorly at smaller spatial scales. We have shown here that coarse-resolution GCM forecasts can be made more useful at regional or smaller scales through some form of correction for model biases. Statistical downscaling is simpler and cheaper relative to dynamical downscaling but can provide substantial improvements in model performance. The continued use of linear statistical models along with recalibration methods seems an appropriate strategy for deriving seasonal climate forecasts for the SADC region.

\section{ACKNOWLEDGEMENTS}

The Southern African Development Community (SADC) Drought Monitoring Centre, Harare (DMC-H) provided the station rainfall data. Special thanks are expressed to Emmanuel Dlamini for facilitating the first author's attachment to the DMC-H. We are grateful to Emily Grover-Kopec for helping with the extraction of the ECHAM4.5 GCM and SST data from the International Research Institute for Climate and Society (IRI) data library. We greatly appreciate contributions by Anna Bartman (SAWS), Mduduzi Gamedze (Swazi Met.), Henk van den Brink (KNMI) and constructive comments by two anonymous reviewers.

\section{REFERENCES}

Barnett TP, Preisendorfer R. 1987. Origins and levels of monthly and seasonal forecast skill for United States surface air temperatures determined by canonical correlation analysis. Monthly Weather Review 115: 1825-1850.

Barnston AG, Thiaw W, Kumar V. 1996. Long-lead forecasts of seasonal precipitation in Africa using CCA. Weather And Forecasting 11: $506-520$.

Busuioc A, von Storch H, Schnur R. 1999. Verification of GCM generated regional seasonal precipitation for current climate and of statistical downscaling estimates under changing climate conditions. Journal of Climate 12: 258-272.

Busuioc A, Deliang C, Hellström C. 2001. Performance of statistical downscaling models in GCM validation and regional climate change estimates: Application for Swedish precipitation. International Journal of Climatology 21: 557-578.

Gershunov A, Barnett TP. 2003. Interdecadal modulation of ENSO teleconnections. Bulletin of the American Meteorological Society 79: $2715-2725$.

Goddard L, Mason SJ, Zebiak SE, Ropelewski CF, Basher R, Cane MA. 2001. Current approaches to seasonal-to-interannual climate predictions. International Journal of Climatology 21: 1111-1152.

Hastenrath S, Greischar L, van Heerden J. 1995. Prediction of summer rainfall over South Africa. Journal of Climate 8: 1511-1518. 
Jackson JE. 1991. A User's Guide to Principal Components. Wiley: Hoboken, New Jersey, USA, 569.

Joubert AM. 1997. Simulations by the atmospheric model intercomparison project of atmospheric circulation over southern Africa International Journal of Climatology 17: 1129-1154.

Karl TR, Wang WC, Schlesinger ME, Knight RW, Portman D. 1990. A method of relating general circulation model simulated climate to the observed local climate. Part I: Seasonal statistics. Journal of Climate 3: 1053-1079.

Kidson JW, Thompson CS. 1998. A comparison of statistical and model-based downscaling techniques for estimating local climate variations. Journal of Climate 11: 735-753.

Landman WA, Mason SJ. 1999a. Change in the association between Indian ocean sea-surface temperatures and summer rainfall over South Africa and Namibia. International Journal of Climatology 19: 1477-1492.

Landman WA, Mason SJ. 1999b. Operational long-lead prediction of South African rainfall using canonical correlation analysis. International Journal of Climatology 19: 1073-1090.

Landman WA, Tennant WJ. 2000. Statistical downscaling of monthly forecasts. International Journal of Climatology 20: $1521-1532$.

Landman WA, Goddard L. 2002. Statistical recalibration of GCM forecasts over southern Africa using model output statistics. Journal of Climate 15: 2038-2055.

Landman WA, Mason SJ, Tyson PD, Tennant WJ. 2001. Retroactive skill of multi- tiered forecasts of summer rainfall over southern Africa. International Journal of Climatology 21: 1-19.

Makarau A, Jury MR. 1997. Predictability of Zimbabwe summer rainfall. International Journal of Climatology 17: $1421-1432$.

Mason IT. 2003. Binary events. In Forecast Verification: A Practitioner's Guide in Atmospheric Science, Jolliffe IT, Stephenson DB (eds). Wiley: Chichester, West Sussex, England, 37-76.

Mason SJ. 1998. Seasonal forecasting of South African rainfall using a non-linear discriminant analysis model. International Journal of Climatology 18: 147-164.

Mason SJ. 2004. On using "climatology" as a reference strategy in the Brier and ranked probability skill scores. Monthly Weather Review 137: 1891-1985.

Mason SJ, Graham NE. 1999. Conditional probabilities, relative operating characteristics, and relative operating levels. Weather and Forecasting 14: 713-725.

Mason SJ, Mimmack GM. 2002. Comparison of some statistical methods of probabilistic forecasting of ENSO. Journal of Climate 15: $8-29$.

Mason SJ, Joubert AM, Cosijn C, Crimp SJ. 1996. Review of seasonal forecasting techniques and their applicability over southern Africa. Water Sa 22: 203-209.

Mimmack GM, Mason SJ, Galpin JS. 2001. Choice of distance matrices in cluster analysis: defining regions. Journal of Climate 14: $2790-2797$.

Mo R, Stratus DM. 2002. Statistical-dynamical seasonal prediction based on principal components regression of GCM ensemble integrations. Monthly Weather Review 130: 2167-2187.

Murphy J. 1999. An evaluation of statistical and dynamical techniques for downscaling local climate. Journal of Climate 12: 2256-2284.

Murphy J. 2000. Predictions of climate change over Europe using statistical and dynamical downscaling techniques. International Journal of Climatology 20: 489-501.

Nicholson SE, Kim J. 1997. The relationship of the El Nino Southern oscillation to African rainfall. International Journal of Climatology 17: $117-135$.

Rocha A, Simmonds I. 1997. Interannual variability of south-eastern African summer rainfall. Part II: modelling the impact of sea-surface temperatures on rainfall and circulation. International Journal of Climatology 17: 267-290.

Roeckner E, Arpe K, Bengtsson L, Claussen CM, Dmenil L, Esch M, Giorgetta M, Schiese U, Schulzweida U. 1996. The atmospheric general circulation model ECHAM-4: model description and simulation of present-day climate. Max-Planck-Institute for Meteorology Report No. 218, Hamburg, Germany.

Ropelewski CF, Halpert MS. 1987. Global and regional scale precipitation patterns associated with El Ni no/Southern Oscillation. Monthly Weather Review 115: 1606-1626.

Smith TM, Reynolds RW. 2004. Improved extended reconstruction of SST (1854-1997). Journal of Climate 17: $2466-2477$.

Tabachnick BG, Fidell LS. 2001. Using Multivariate Statistics, 4th edn. Allyn and Bacon: Needham Heights, USA, 966.

Tennant W. 1999. Numerical forecasting of monthly climate in South Africa. International Journal of Climatology 19: 1319-1336.

Toth Z, Talagrand O, Candille G, Zhu Y. 2003. Probability and ensemble forecasts. Forecast Verification: A Practitioner's Guide in Atmospheric Science, Jolliffe IT, Stephenson DB (eds). Wiley: Chichester, West Sussex, England, 137-163.

Unganai LS, Mason SJ. 2002. Long-range predictability of Zimbabwe summer rainfall. International Journal of Climatology 22: $1091-1103$.

van Oldenborgh GJ, Balmaseda MA, Ferranti L, Stockdale TN, Anderson DLT. 2005. Evaluation of atmospheric fields from the ECMWF seasonal forecasts over a 15-year period. Journal of Climate 18: 3250-3269.

von Storch H, Zwiers FW. 1999. Statistical Analysis in Climate Research. Cambridge University press: Cambridge, United Kingdom, 484.

von Storch H, Zorita E, Cubash U. 1993. Downscaling of global climate change estimates to regional scale: an application to Iberian rainfall in wintertime. Journal of Climate 6: 1161-1171.

Wilks DS. 1995. Statistical Methods in the Atmospheric Sciences. Academic press: San Diego, California, USA, 467.

Yu Z-P, Chu P-S, Schroeder T. 1997. Predictive skills of seasonal to annual rainfall variations in the U.S. affiliated Pacific islands: Canonical correlation analysis and multivariate principal component regression approaches. Journal of Climate 10: 2586 -2599.

Zorita E, von Storch H. 1999. The analog method as a simple statistical downscaling technique: comparison with more complicated methods. Journal of Climate 12: 2474-2489. 